\title{
REDUCTION OF RF SKIN LOSS WITH THIN FOILS
}

\author{
Y. Iwashita, H. Fujisawa, M. Ichikawa, Y. Tajima, Kyoto ICR, Uji, Kyoto, Japan
}

\section{Abstract}

Reduction of RF power loss caused by skin effect has been studied. Some measurement results on a coaxial cavity with thin foils are described.

\section{INTRODUCTION}

RF wall loss caused by skin effect has been a common sense to those who are working on RF devices such as cavities or transmission lines. Although reduction the surface loss was suggested by A. M. Clogston in 1951 [1], such a technique has not been widely used in the field. Recently, it is shown that such a power loss can be practically reduced on a dielectric resonator with thin conductor layers on the surface, where the layers are thinner than the skin depth [2]. There is a restriction on the dielectric constant of the insulating layers, while such a restriction was reduced by shaping the conductor layers [3]. Preliminary experiments of the reduction of the skin loss on a cavity surface (not a dielectric material filled resonator) was performed by changing the insulator thickness along the current direction and reported in ref.4. This paper describes a subsequent precise experiment and simulation results, followed by some reviews.

\section{SKIN EFFECT ON CONDUCTORS}

Suppose a system as shown in Fig. 1 where the conductor has conductance $\sigma(\gg>i \omega \varepsilon)$, the current distribution is expressed by:

$$
j(x)=j_{0} e^{-(1+i) x / \delta}, \quad \delta=\sqrt{2 / \omega \mu \sigma},
$$

$\delta$ is the so-called skin depth.

Let us consider a case that the thickness of the conductor is thinner than the skin depth $\delta$ (see Fig. 2) and the both sides have electromagnetic fields with different amplitudes. The current distribution becomes:

$$
\begin{aligned}
& j(x)=H_{z}(0)\left(j_{f} e^{-(1+i) x / \delta}+j_{b} e^{-(1+i)(\alpha \delta-x) / \delta}\right), \\
& j_{f}=\frac{(1+i) e^{(1+i) \alpha}\left(e^{(1+i) \alpha}-\xi\right)}{\delta\left(e^{2(1+i) \alpha}-1\right)}, j_{b}=\frac{(1+i) e^{(1+i) \alpha}\left(\xi e^{(1+i) \alpha}-1\right)}{\delta\left(e^{2(1+i) \alpha}-1\right)} .
\end{aligned}
$$

For a case when the magnetic fields of both sides have the same amplitude $(\xi=1)$, the current is zero at the origin because the currents from both sides cancel each other.

Let us consider a simple example as shown in Fig. 3(b), where a layer of thin conductor foil with thickness of about the skin depth $\delta$ covers the conductor surface. The total current of the thin layer is just the magnetic field difference between the front and backsides. When the magnetic field ratio $\xi$ is properly chosen, the currents are well distributed wider than just skin depth $\delta$. For example, when $\alpha=0.75$ and $\xi=0.6$, the total power loss becomes about $70 \%$ of the bulk case (Fig. 4).

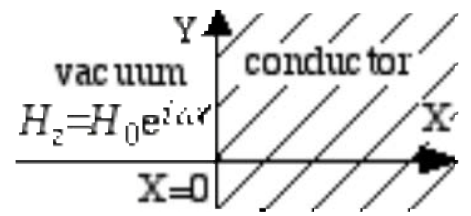

Figure 1: Half of the space is filled by conductor.

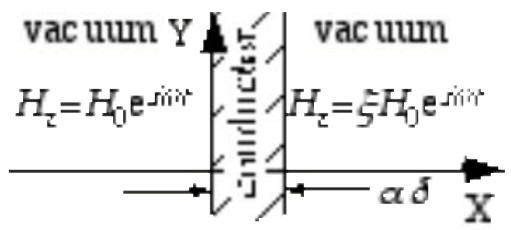

Figure 2: The thickness is thinner than the skin depth.

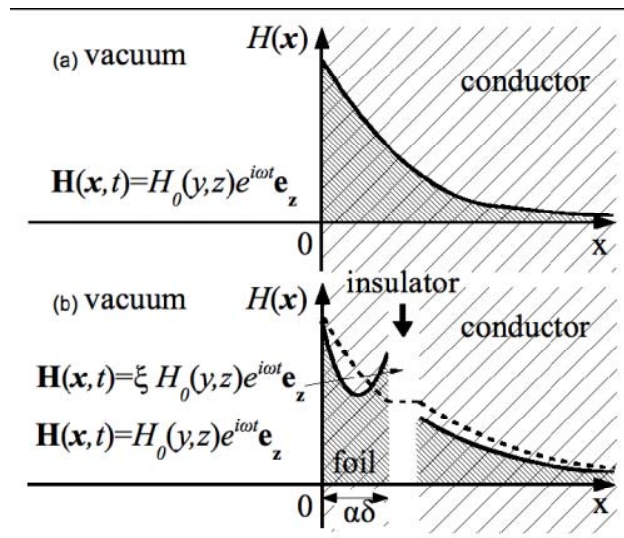

Figure 3: Bulk conductor and a conductor foil immersed in RF field. The magnetic field has only a component parallel to the surface of the bulk and the foil. (a) A bulk conductor alone. (b) An insulated conductor foil layer on a bulk conductor. The solid lines shown in the conductors shows the absolute value of the current density or the magnetic field in the conductors. The broken line shows that in the bulk case (a) as a comparison.

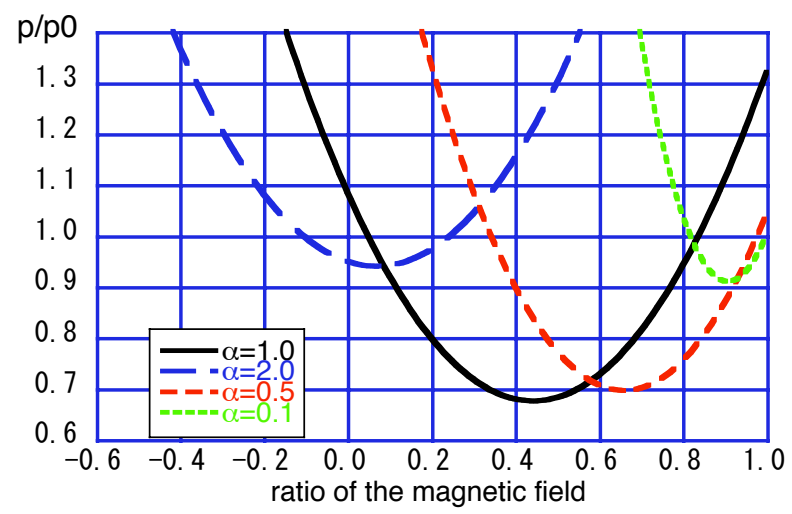

Figure 4: The power loss $\mathrm{P}$ of Fig. 3(b). The power loss is normalized by $\mathrm{P} / \mathrm{P} 0$. P0 is the power loss of Fig. 3(a) 


\section{DIELECTRIC RESONATOR}

Let us consider a flat dielectric cylindrical resonator of $\mathrm{TM}_{010}$ mode with high dielectric constant as shown in Fig. 5. The current along the radius has the anti-node at the middle of the radius.

When we put extra thin washer shape foils at the peak area as shown in Fig. 6, part of the displacement currents go into the extra thin foils and flow in them, which redistributes the current flows. It should be noted that the space between the disc and the extra electrodes are essential; it should have lower dielectric constant than the body material. As can be seen, the width of the extra electrode is comparable to the half wavelength in the main body. Therefore the narrow space between two electrodes (not the wider one that forms the resonator itself) becomes another resonator to suck the power unless the space has enough lower dielectric constant than that of main body.

\section{LOCAL RESONANCE CONTROL}

The scheme works well because the dielectric constant of the resonator body is much higher than that of the insulating layer. In other words, the wavelength in the insulating layer is longer than that of the body, and thus the resonant frequency of the insulating space is far from the operating frequency. For a cavity whose body has the dielectric constant of unity, it is difficult for the dielectric constant of the insulating layer to have such a condition: dielectric constant of less than unity.

Because the principal requirement of the method is that the local resonant frequency of the insulating space should be far enough not to have extra energy in it. Fig. 7 (left) shows the schematic picture of such a local resonance model. This structure acts as a $\lambda / 2$ mode resonator when a condition meets; electric and magnetic field distributions have a node and an antinode at the center, respectively. This can be described by an equivalent circuit model as shown in Fig. 8 (right). The local resonant frequency can be raised by reducing $\mathrm{L}$ and/or $\mathrm{C}$ as shown in Fig.8: to widen the gap wider at the edge areas and decrease it at the center area.

\section{MEASUREMENT ON COAXIAL CAVITY}

The effect was experimentally tested on a coaxial cavity, because it has a simple electromagnetic field distribution (see Fig.9). A thin film ( $5 \mu \mathrm{m}$ copper layer on $25 \mu \mathrm{m}$ polyimide [5]) was put on the inner conductor with its polyimide side down to form an insulation layer. Because the film has to be located at the high magnetic field region, the resonant mode for the measurement was the second lowest mode that has an antinode of its magnetic field at the center; other antinode regions are located at the ends and are difficult to put the films. The film locally covers the center inner region and the $\mathrm{Q}$ values were measured while changing the length by cutting from its edges step by step. The length of the film can control the total current on the film that goes in and out as a displacement current.

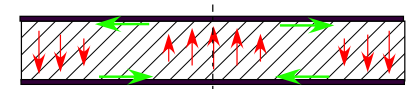

Figure 5: Flat dielectric cylindrical cavity where the dielectric constant $\varepsilon_{\mathrm{r}}$ is much larger than unity and the top and the bottom surfaces are covered by conductors.

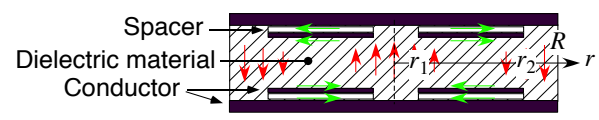

Figure 6: Dielectric cylindrical cavity with extra electrodes that have washer shape. The thicknesses are exaggerated: inside of the each conventional disc electrode, one smaller washer shape electrode is located with a small distance from the disc.

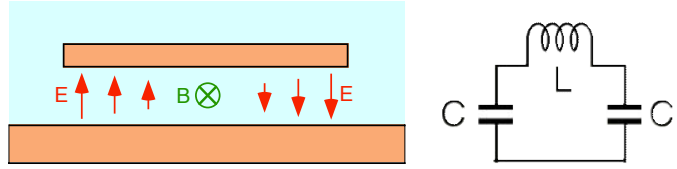

Figure 7: Left: local resonance at inter layer space and Right: its equivalent circuit

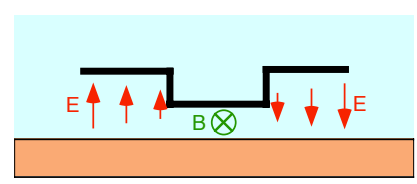

Figure 8: Raised local resonant frequency.

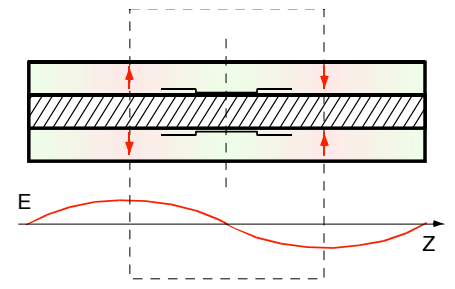

Figure 9: Experiment on a coaxial cavity.

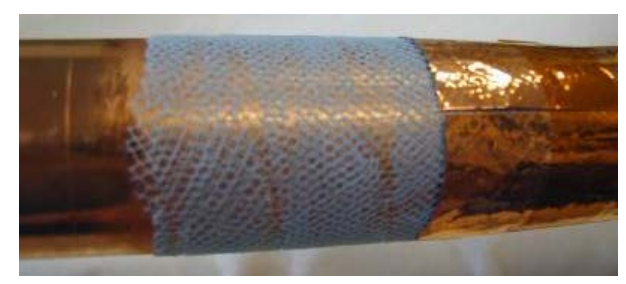

Figure 10: Polyethylene mesh as an extra spacer.

The cavity has length of $2 \mathrm{~m}$ to have its second lowest frequency $150 \mathrm{MHz}(\lambda=2 \mathrm{~m})$. The skin depth of the copper conductor at this frequency is $5.4 \mu \mathrm{m}$. $80 \mathrm{~cm}$ of the center region is initially wrapped around where the film was put directly on the inner most $30 \mathrm{~cm}$ region while the outer areas have spacer layers by putting polyethylene meshes with $300 \mu \mathrm{m}$ thickness (see Figure 10). The Q-value was measured while the edges of the film were shortened. The measured result is shown in Figure 11 together with a calculation result. The ordinate shows a ratio $\mathrm{Q} / \mathrm{Q}_{0}$, where $\mathrm{Q}_{0}$ is the measured Q-value when all the film was removed lastly. The abscissa shows a length of the 
covered length by film to $\lambda / 2$. Because the observed frequency change was less than $10^{-3}$ and the volume under the conductor foil is very small compared with the cavity body, the electromagnetic field distribution should not have changed very much. The value of more than unity shows that the real power loss reduction was achieved with this scheme. The calculations were based on a configuration shown in Fig.12 using CFISH, which is a part of POISSON/SUPERFISH code group. It treats whole conductor surface as a lossy material, which tends to become very large problem because of the very small mesh size in conductor area compared with the total size. The results agreed with the measured data, considering the difficulty in setting the film on the inner conductor with the polyethylene mesh.

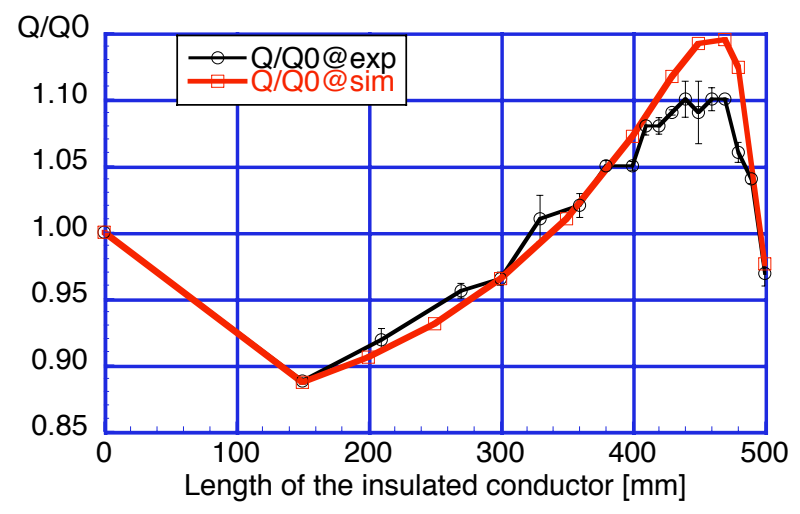

Figure 11: Measured and calculated $Q$ values

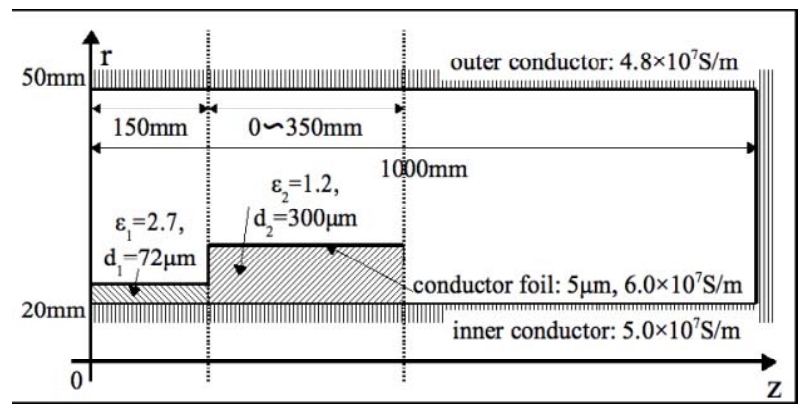

Figure 12: Geometry for the $\mathrm{Q}$ value estimation.

\section{TRANSMISSION LINE ANALYSIS}

The results were analyzed by transmission line theory. While details will be published soon, brief procedure will be explained here. The bulk conductor and the foil form a transmission line. In the case discussed above, the impedance between the two conductors can be evaluated along the axis, once the capacitance and inductance per unit length are known along the axis, where the characteristic impedance is known. As shown in Fig. 13, the impedance is zero at the position $z=0$ because of the symmetry. The magnetic field has its antinode here. Then the impedance goes up along the axis and the spatial phase advances. If the phase advance reaches 90 degree at the end, the impedance goes to infinity and the

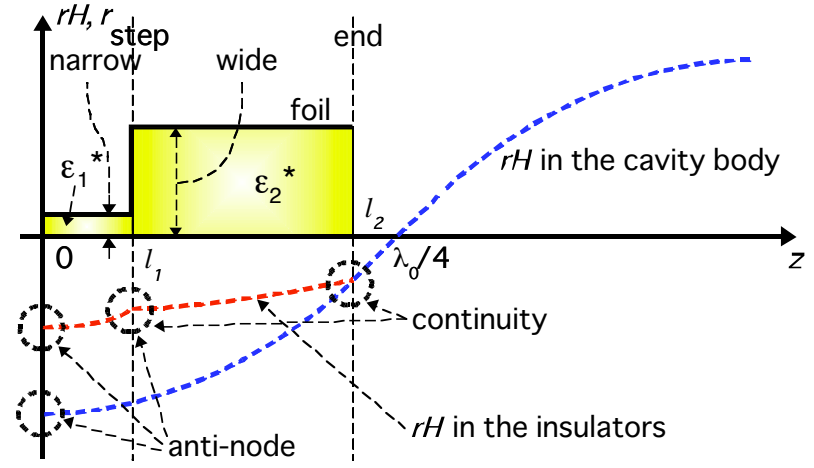

Figure 13 The distribution of the magnetic field, $\mathrm{rH}$, when the insulated conductor has a stepped structure.

transmission line resonates at the frequency. This situation should be avoided because it sucks much energy. When the thickness of the insulating layer becomes large at $\mathrm{z}=l_{1}$, the characteristic impedance jumps up. When we take a look at the impedance from the wider region, the impedance of the narrower area is very small and looks like a short-circuit. The spatial phase is practically reset by this effect and starts over from almost zero degree. Consequently, the total length of the foil can be long with less phase advance at the end $\left(\mathrm{z}=l_{2}\right)$. In other words, the resonant frequency of the region can be raised

\section{DISCUSSIONS}

Reducing RF losses of waveguide and accelerating cavity are one of the final goals of this study. Although the restriction on the permittivity can be reduced, only the stepping configuration is not enough to achieve the enhancement yet, because these RF objects have longer wall distances compared with the wavelength. More sophisticated idea is needed for these kind of RF objects.

\section{ACKNOWLEDGEMENT}

The author would like to thank Mr. H.Tongu for his technical help in measuring the $\mathrm{Q}$ value.

\section{REFERENCES}

[1] A. M. Clogston, Reduction of Skin-Effect Losses by the Use of Laminated Conductors, Proc. of the IRE, 39-7, July 1951, pp.767-782

[2] J. Hattori, et al.: Low Profile Dielectric Band Elimination Filter using Thin Film Layered Electrode for $2 \mathrm{GHz}$ Band Cellular Base Station, IEEE MTT-S Digest 1999

[3] Y. Iwashita:Reduction of Power Loss Caused by Skin Effect, LINAC 2004, Lübeck, Germany pp. 700-702. http://cern.ch/AccelConf/104/PAPERS/THP43.PDF

[4] Y. Iwashita: "Mitigation of Power Loss Due to Skin Effect by Thin-Layered Film" ,Proc. of LINAC 2006, Knoxville, TN, USA pp. 785-787 http://cern.ch/AccelConf/106/PAPERS/THP086.PDF

[5] http://www.toray-taf.co.jp/img/product_group/ Metaloyal.pdf, in Japanese 